\title{
Trajectory binning scheme and non-active treatment of zero-point energy leakage in quasi-classical dynamics
}

\author{
A.J.C. Varandas * \\ Departamento de Química, Universidade de Coimbra, 3004-535 Coimbra, Portugal
}

Received 15 January 2007; in final form 19 March 2007

Available online 30 March 2007

\begin{abstract}
By expressing an unknown state in terms of a complete set, a simple scheme for approximate quantization of the continuous vibrational-rotational energy distributions that are obtained from quasi-classical trajectory calculations is suggested. The problem of zeropoint energy leakage is also revisited, and the new method tested on the prototype $\mathrm{O}+\mathrm{OH}$ and $\mathrm{H}+\mathrm{D}_{2}$ reactions.
\end{abstract}

(C) 2007 Elsevier B.V. All rights reserved.

\section{Introduction}

The quasi-classical trajectory (QCT) method is by far the most popular tool for studying molecular reaction dynamics. Although quantum mechanical (QM) effects are not taken into account (except for the fact that vibrations and rotations of the reagents are quantized at the initial instant when integrating the equations of motion), one requires for a detailed comparison with experiment that the vibrational and rotational states of the products are quantized. The traditional approach consists of quantizing the rotational and vibrational components of the internal energy by using the histogram method to obtain approximate integer rotational and vibrational quantum numbers. Of course, limiting the statistics to trajectories leading to true integer vib-rotational actions would be helpless as they form a set of measure zero. To obtain an improved vibrational-rotational distribution for $\mathrm{AB}(v, j)+\mathrm{C}$ products, Bonnet and Rayez [1] (BR) suggested to weight each trajectory by

$C_{v j}^{i}=\exp \left\{\left[\left(v-x_{v}^{i}\right)^{2}+\left(j-x_{j}^{i}\right)^{2}\right] / \epsilon^{2}\right\}$

\footnotetext{
${ }^{*}$ Fax: + 351239835867.

E-mail address: varandas@qtvs1.qui.uc.pt
}

where $i$ is the trajectory number, $v$ and $j$ are the vibrational and rotational quantum numbers to which the trajectory is supposed to contribute, $x_{v}^{i}$ and $x_{j}^{i}$ are the vibrational and rotational actions obtained through partition of the internal energy of the products, and $\epsilon$ is an arbitrary parameter with a value much lower than 1 . The corrected state distribution assumes the form $P_{v j}=\sum_{i} C_{v j}^{i}$, with all trajectories taken into account. Since the number of rotational states usually exceeds by far the vibrational one, BR actually considered the rotational angular momentum as continuous by limiting quantization to the vibrational motion. Although BR rationalized [2] their approach with basis on classical $S$-matrix theory in the random-phase approximation, an alternative rationale would be desirable as the former is applicable to situations where the density of states is supposedly large and hence favorable to application of the traditional binning scheme.

In this work, we follow the spirit of the BR proposal (see also Bañares et al. [3] for an equivalent procedure that has been employed to study the $\mathrm{C}\left({ }^{1} \mathrm{D}\right)+\mathrm{H}_{2}$ reaction) but use an alternative approach which is tested on both indirect and direct-type prototype reactions, respectively $\mathrm{O}+\mathrm{OH}$ and $\mathrm{H}+\mathrm{D}_{2}$. In relation to binning, we discuss also the so-called zero-point energy (ZPE) leakage problem of classical mechanics which is due to the flow of vibrational energy (this cannot be smaller than ZPE in QM) into other degrees of freedom (Ref. [4], and references therein). 
The Letter is structured as follows. Section 2 describes the method, which is then tested and the results discussed in Section 3. Section 4 gathers the conclusions.

\section{Binning procedure}

Rather than using vibrational and rotational quantum numbers obtained via the traditional histogram binning procedure, we employ directly the energies obtained from partition of the internal energy. As it is well known, this procedure is not error-free due to the non-harmonic nature of the potential energy surface (PES) which poses a conceptual difficulty to the separability of the internal energy. However, we will follow common wisdom and do not address this issue but for a comment later.

Consider the two-dimensional (2D) grid of vibrational vs rotational $\left(E_{\mathrm{v}}\right.$ vs $\left.E_{\mathrm{r}}\right)$ energies obtained by solving the Schrödinger equation for the potential curve of the product diatomic molecule. It defines a 2D-space where the 'vibrational-rotational state' $\left(\mathscr{E}_{\mathrm{v}}, \mathscr{E}_{\mathrm{r}}\right)$ extracted from a QCT trajectory maps into a point. Only by accident will such a 'classical state' coincide with a quantum one. It is on the assignment of this state to the available quantum ones that we focus in the present work.

Consider now a particle of mass $m$ moving along the $x$ dimension. The particle wave-packet assumes the form

$\Psi(x, t)=\int_{-\infty}^{+\infty} A(\kappa) \exp (\mathrm{i} \kappa x) \exp (-\mathrm{i} \omega t) \mathrm{d} \kappa$

where $E_{\kappa}=\hbar^{2} \kappa^{2} / 2 m$ is the energy associated to the momentum $p_{\kappa}=\hbar \kappa, \omega_{\kappa}=E_{\kappa} / \hbar$, and $\hbar$ is the Planck constant divided by $2 \pi$. The particle can therefore be viewed as a wave-packet constructed from a broad range of wave numbers $\kappa=2 \pi / \lambda$ so that it becomes highly localized in space. The degree of such a localization is determined by the spectral function $|A(\kappa)|^{2}$ that defines the contributing probability of the wave number $\kappa=2 \pi / \lambda$ (or its associated momentum $p_{k}$ ) to the particle wave-packet. Of course, the wave-packet evolution in time corresponds to the particle trajectory.

A general form of spectral function is the wave-packet of minimum width (Gaussian wave-packet):

$A(\kappa)=\exp \left[-c^{2}\left(\kappa-\kappa_{0}\right)^{2}\right]$

where $c$ is a constant. The center of the wave-packet $\langle x\rangle$ at any time $t$ is given by [5] $\hbar \kappa_{0} t / m$, and hence $p_{\kappa_{0}}=\hbar \kappa_{0}$ is the classical momentum of the particle. It is also well known that the wave-packet spreads in $x$ as time evolves, with spreading occurring for any wave-packet and even the shape changing in most wave-packets. However, in the case of a Gaussian wave-packet, its shape remains Gaussian but the width increases with time. This spreading may be thought of as due to the fact that the waves of different frequencies $\omega_{\kappa}$ move with different velocities, and hence gradually begin to separate as time progresses. We emphasize the Gaussian shape of the momentum distribution which is centered at $p_{\kappa_{0}}=\sqrt{2 m E_{\kappa_{0}}}$. It is on a generalization of this premise that we base our scheme, hereafter called momentum Gaussian binning (MGB), to quantize the classical vibrational-rotational energy distribution of the product diatomic $\mathrm{AB}$ and hence justify the Gaussian weighting of trajectories in the QCT method.

Consider then the separating $\mathrm{AB}-\mathrm{C}$ reaction products in the ith trajectory. They have associated a particle wave-packet that may be thought of as composed of two wave-packets. One describes the translational motion of the system as a whole, the other describes the internal vibrational-rotational motion of $\mathrm{AB}$. This may itself be thought of as composed of two Gaussian momentum distributions as in Eq. (3): one for vibration, the other for rotation. It is on these distributions that we concentrate next. To define them, we consider the kinetic energies associated to the vibrational and rotational motions. For the former, the arguments invoked in the preceding paragraph suggest a Gaussian dependence on the momentum of the vibrating diatom. Since $E_{\kappa}=\left(v+\frac{1}{2}\right) \hbar \omega_{\kappa}$ for a harmonic oscillator, one then expects a Gaussian dependence in the displacements $\left(\sqrt{v}-\sqrt{v_{0}^{i}}\right)$ where $v_{0}^{i} \hbar$ is the classical vibrational action obtained from trajectory calculations, rather than a Gaussian dependence in $\left(v-v_{0}^{i}\right)$ as utilized by BR [1]. In turn, for a rigid rotor, the classical rotational energy is given by $E_{\kappa}=\left(I \omega_{\kappa}\right)^{2} / 2 I$, where $\mathscr{J}_{\kappa}=I \omega_{\kappa}$ is the angular momentum and $I$ the moment of inertia. Thus, the MGB method predicts a Gaussian dependence in the displacements $\left(\mathscr{J}_{\kappa}-\mathscr{J}_{0}^{i}\right)$ or, using the well known quantization rule $\mathscr{J}=[j(j+1)]^{1 / 2} \hbar \propto j \hbar$, in the displacements $\left(j-j_{0}^{i}\right)$ where $j_{0}^{i} \hbar$ is the classical rotational action obtained from trajectory $i$, as prescribed by BR [1].

Let us now denote the wave-packet associated to an arbitrary trajectory $i$ by $\Psi_{v j}^{i}$. Obviously, if known, such a wave-packet could be projected into the asymptotic product vibrational-rotational states $\left\{\phi_{\alpha \beta}\right\}$ to generate the populations. One has

$\Psi_{v j}^{i}=\sum_{\alpha \beta} c_{\alpha \beta}^{i} \phi_{\alpha \beta}$

and, by considering the vibrational-rotational Hamiltonian $(\mathscr{H})$ of the product diatomic

$\left\langle\Psi_{v j}^{i}|\mathscr{H}| \Psi^{i}{ }_{v j}\right\rangle=\sum_{\alpha \beta} W_{\alpha \beta}^{i}\left\langle\phi_{\alpha \beta}|\mathscr{H}| \phi_{\alpha \beta}\right\rangle$

where $W_{\alpha \beta}^{i}=c_{\alpha \beta}^{i \star} c_{\alpha \beta}^{i}$, and the basis functions are assumed to form an orthonormal set. The vibrational-rotational energy of such a wave-packet may then be expressed as

$\mathscr{E}_{v j}^{i}=\sum_{\alpha \beta} W_{\alpha \beta}^{i} E_{\alpha \beta}$

where $W^{i}$ is a $2 \mathrm{D}$ probability matrix. Note that $W^{i}$ cannot be determined from $\mathscr{E}_{v j}^{i}$, as it would imply knowing all coefficients

$c_{\alpha \beta}^{i}=\left\langle\phi_{\alpha \beta} \mid \Psi_{v j}^{i}\right\rangle$

which is obviously impossible due to not knowing the wave-packet $\Psi_{v j}^{i}$. Some approximate scheme will then be 
mandatory. Following Eq. (3), the weights $W_{\alpha \beta}^{i}$ should reflect a squared Gaussian dependence on the displacements from the square-root of the energy of a given contributing state to the supposedly known classical value (viewed as the centroid of the relevant quantum distribution). Assuming separability of the vibrational and rotational degrees of freedom, one gets

$W_{\alpha \beta}^{i}=W_{\alpha}^{i} W_{\beta}^{i}$

where

$W_{\alpha}^{i}=\frac{1}{\rho_{\alpha} \sqrt{\pi}} \exp \left[-\left(\frac{\sqrt{E_{\alpha}}-\sqrt{\mathscr{E}_{\alpha}^{i}}}{\rho_{\alpha} \sqrt{\bar{E}_{\alpha}}}\right)^{2}\right]$

defines the Gaussian-type vibrational distribution, and a corresponding expression holds for the $\beta$ rotational states. Note that $\bar{E}_{\alpha}$ is an average energy separation of two neighboring vibrational states of the basis as obtained from

$\bar{E}_{\alpha}=\left(E_{\alpha+1}-E_{\alpha-1}\right) / 2$

such that $\rho_{\alpha}$ becomes a unitless Gaussian decay parameter. If $\alpha$ refers to the first (last) vibrational state, Eq. (10) is replaced by $\bar{E}_{\alpha}=\frac{1}{2}\left(E_{\alpha} \pm E_{\alpha \pm 2}\right)$, with the plus (minus) sign applying to the former (latter) case. Corresponding definitions hold for rotation.

Some remarks on the separability of vibrational and rotational motions and use of simple harmonic oscillatorrigid rotor formulas are in order at this point. The former guided on establishing Eq. (9) from Eq. (3), while the latter helped rationalizing the Gaussian weighting of trajectories in the QCT method. These bear no limitations whatsoever on the formalism, with $\rho_{\alpha}$ and $\rho_{\beta}$ acting as fine-tuning Gaussian-decay parameters for further realism. In fact, the method compares rigorous QM vibrational and rotational energies with their classical counterparts, and hence makes no approximation except for treating them as commonly done (i.e., separately). This premise could be released by considering a vector of vib-rotational energies in Eq. (8) and an adequate replacement for Eq. (9), an approach that we hope to explore in future work.

Of the Gaussian decay parameters $\rho_{\alpha}$ and $\rho_{\beta}$, the former dominates in Eq. (8), and will be considered first. In so doing, we aim also at an approximate non-active scheme that accounts for ZPE leakage. For example, if $\rho_{\alpha}=0.1$, the contribution to the ground-state vibrational population $W_{0}^{i}$ of a trajectory ending with a vibrational energy $\mathscr{E}_{\mathrm{v}}=0.9 E_{0}$ turns out to be about $48 \%$ of the value that would actually be obtained if $\mathscr{E}=E_{0}$. Similarly, for values of $\mathscr{E}_{\mathrm{v}}=0.5 E_{0}$ and $0.1 E_{0}$, the corresponding results would be $1.6 \%$ and $0.006 \%$. Such values appear reasonable when judged from physical intuition, and so $\rho_{\alpha}$ has been fixed at the above value. In turn, the determination of $\rho_{\beta}$ was set from the observation that, for the energy values usually encountered in chemistry, the rotational spacing is typically much smaller than the vibrational one. As a result, a more accentuated Gaussian decay is expected, i.e., $\rho_{\beta} / \rho_{\alpha}>1$.
Thus, we have fixed it from the ratio of the averaged square-root vibrational and rotational spacings, with the latter estimated by considering all vibrational states. This led to $\rho_{\beta}=0.172$ and 0.202 for the $\mathrm{O}+\mathrm{OH}$ and $\mathrm{H}+\mathrm{D}_{2}$ reactions, respectively. Of course, $\rho_{\alpha}$ and $\rho_{\beta}$ could be taken as adjustable parameters to reproduce any known QM result, although this approach will not be pursued in the current work. Other binning details that are specific of the systems here studied will be deferred to Section 3 .

As noted in the Introduction, all trajectories contribute to all open vib-rotational states, although only a few are expected to do so in a significant manner. Thus, unlike other non-active models [6,7], no trajectories are discarded from the statistical analysis when accounting for ZPE leakage. The approach, thereafter referred to as QCT/momentum Gaussian-binning (QCT/MGB or shortly MGB) method, shows similarities with running wave-packets as all energetically accessible states of the products receive some scattering probability. For a given vib-rotational state, the probability estimated from the classical internal energy distribution is given by

$P_{\alpha \beta}=\sum_{i=1}^{N} W_{\alpha \beta}^{i}$

where the summation is over the whole set of trajectories (in a total of $N$ ) that contribute to the particular set of products under consideration.

\section{Results and discussion}

The MGB method here reported has been tested on the $\mathrm{O}+\mathrm{OH}(v, j)$ and $\mathrm{H}+\mathrm{D}_{2}(v=0, j=0)$ reactions. All calculations employed the VENUS96 trajectory code [8], and reliable double many-body expansion [9] (DMBE) PESs. With impact parameters optimized to within $0.1 a_{0}$ or so, batches of $10^{5}$ trajectories have been run in both cases, warranting conservation of total energy to better than 1 part in $10^{5}$. For $\mathrm{O}+\mathrm{OH}(v, j)$, we have employed method I (i.e., a single impact parameter) of Ref. [10].

\subsection{The $\mathrm{O}+\mathrm{OH}$ reaction under local thermodynamic disequilibrium}

The hydrogen-oxygen systems play a key role in the chemistry of Earth's atmosphere, combustion, and laser processes [4]. Specifically, the $\mathrm{O}+\mathrm{OH}$ reaction is the reverse of the most important reaction in combustion. It is also a prototype for a barrier-free reaction dominated by long range forces, with the trajectories being largely trapped in the involved deep potential well of $\mathrm{HO}_{2}$ as one expects for an indirect-type reaction. In turn, the quenching of vibrationally excited $\mathrm{OH}$ by oxygen atoms is critical for establishing the populations of the various vibrational states and hence for modeling the $\mathrm{OH}$ nightglow in the middle atmosphere [11]. Recently [10], calculations have been reported of the reaction $\mathrm{O}+\mathrm{OH}(v, j) \rightarrow \mathrm{O}_{2}\left(v^{\prime}, j^{\prime}\right)+\mathrm{H}$ (see 
Ref. [12] for a similar study of the $\mathrm{O}+\mathrm{HO}_{2}$ reaction) with vibrationally hot reactants, aiming at examining the implications of local thermodynamic disequilibrium (LTD) in atmospheric modeling.

All calculations employed the DMBE IV [13] PES for $\mathrm{HO}_{2}\left({ }^{2} A^{\prime \prime}\right)$, with the collision energy $\left(E_{\mathrm{tr}}\right)$ being selected from a Maxwell-Boltzmann distribution by using the cumulative function

$$
G\left(E_{\mathrm{tr}}\right)=\left(\frac{1}{k_{\mathrm{B}} T}\right)^{2} \int_{0}^{E_{\mathrm{tr}}} E_{\mathrm{tr}}^{\prime} \exp \left(-E_{\mathrm{tr}}^{\prime} / k_{\mathrm{B}} T\right) \mathrm{d} E_{\mathrm{tr}}^{\prime}
$$

where $E_{\mathrm{tr}}$ is chosen randomly for each trajectory by solving $G\left(E_{\text {tr }}\right)-\xi_{1}=0, \xi_{k}(k=1-3)$ is a random number, and $k_{\mathrm{B}}$ the Boltzmann constant. In turn, the $\mathrm{OH}$ vibrational $(v)$ and rotational $(j)$ quantum numbers were sampled from the cumulative distribution function $C(E)=\sum_{n=0}^{v, j} P(n)$, where $P(n)$ may be a pure vibrational distribution or a rotational distribution for a given $v$ state. To simulate a $(v, j)$ state, the summation is first allowed to run from the lowest vibrational level up to the $v$ that satisfies $C\left(E_{\mathrm{v}}\right)-\xi_{2}=0$ and, by using the $v$ value so obtained, carried up to the rotational state $j$ that satisfies $C\left(E_{j, v}\right)-\xi_{3}=0$. Only odd rotational states are allowed, since $\mathrm{OH}$ is considered to belong to Hund's case (b) [14].

The micropopulations used for the calculations are those described elsewhere [10]. Assuming $\mathrm{OH}$ to belong to Hund's case (b), the nuclear rotation quantum number $N$ for the spin-orbit levels $F_{1}$ is given by $N=J+1 / 2$. We use $N$ (denoted $j$ for consistency), and the steady-state rotational distribution [15] that has been obtained from a molecular simulation involving collisions with cold $\mathrm{O}_{2}$ (see Fig. 2 of Ref. [10]). A simulation based on $5 \times 10^{4}$ trajectories has accurately mimicked the initial steady-state distribution giving confidence to the results that are obtained from it.

Since $\mathrm{O}_{2}$ is a Hund's case (b) molecule where only odd numbered rotational states are allowed by symmetry, only odd rotational states are allowed. Similarly, $\mathrm{OH}\left({ }^{2} \Pi\right)$ belongs to Hund's case (b) for a fast rotating $\mathrm{OH}$, and hence the rotational quantum number is given by $j=1,2,3, \ldots$ with the smallest value being $j=\Lambda=1$ where $\Lambda$ is the associated electronic angular momentum. Traditionally [10], the above requirements are satisfied by calculating the rotational state via the expression $j=\frac{1}{2}\left[-1+\sqrt{1+4\left(\frac{L}{\hbar}\right)^{2}}\right]$ and then assigning the final rotational quantum numbers by using $j=\operatorname{INT}(j)+1$ and $j=2 \operatorname{INT}(j / 2)+1$ for $\mathrm{OH}$ and $\mathrm{O}_{2}$, respectively; $L$ is the classical angular momentum of the diatomic, and INT() implies that only the integer part of the argument is retained. Note that the final vibrational quantum numbers $\left(v^{\prime}\right)$ are traditionally obtained by determining first the classical angular momentum of the diatomic via semi-classical quantization followed by rounding-off the calculated real value to its nearest integer. A binning scheme is then followed for the vibrational energy, which is obtained by subtracting the rotational energy from the internal one.
Panel (a) of Fig. 1 shows by the dots the 11588 vibrational-rotational energies associated to the reactive events occurring under LTD at a temperature of $T=255 \mathrm{~K}$. With a maximum impact parameter of $b_{\max }=10.8 \AA$, this yields a rate constant of $k=(36.5 \pm 0.3) \times 10^{-12} \mathrm{~cm}^{3} \mathrm{~s}^{-1}$ as obtained from $k(T)=\langle\mathrm{v}\rangle P_{\mathrm{r}} \pi b_{\max }^{2} ;\langle\mathrm{v}\rangle$ is the average velocity, and $P_{\mathrm{r}}$ the reaction probability at the above temperature. This mimics the result reported elsewhere [10]. Note that $k(T)$ is identical in both QCT and QCT/MGB methods, as the binning procedure can affect only the specific state-to-state rate constants. In turn, panel (a) of Fig. 1 shows by the 'pluses' the 'exact' vibrational-rotational energies of all states (up to the highest populated rotational state in the products) calculated by solving numerically the Schrödinger equation for the $\mathrm{O}_{2}$ potential energy curve using the program LEVEL [16]. Two salient features deserve a comment. First, only by accident does a dot (QCT vibrational-rotational state) matches a plus sign (QM vibrational-rotational state). Since high vibrational excitations are involved, ZPE leakage is small but visible as many dots lie under the lowest row of pluses in
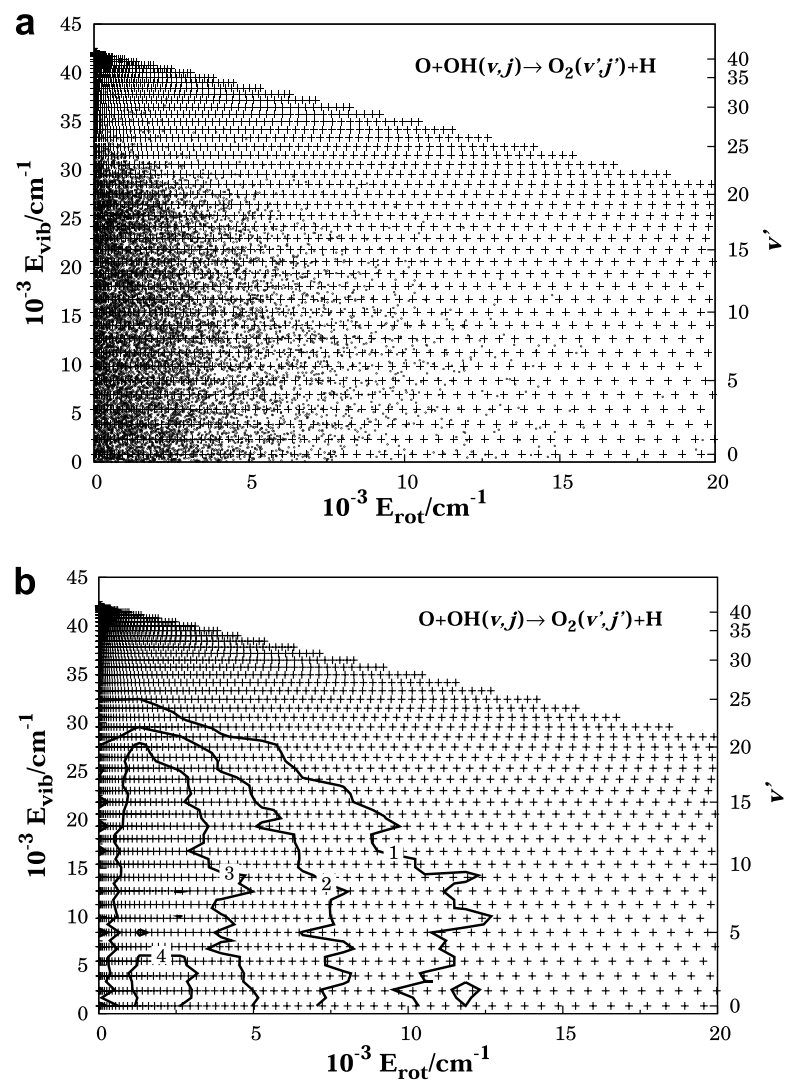

Fig. 1. Panel (a) shows the 2D space defined by the vibrational and rotational energies of the product $\mathrm{O}_{2}$ in the $\mathrm{O}+\mathrm{OH}(v, j)$ reaction under LTD. The 'pluses' indicate the 'exact' vibrational-rotational states while the traditional QCT results (all calculated values are shown) are indicated by the dots. Panel (b) shows a contour plot of iso-probabilities for the vibrational-rotational states of product $\mathrm{O}_{2}$ as given by $P\left(n_{\mathrm{c}}\right)=$ $0.000125 n_{\mathrm{c}}^{2} ; \quad n_{\mathrm{c}}=1,2, \ldots$ (increasing inwards) indicates the contour level. 
Fig. 1a. Superimposed on the grid of exact numerical vibrational-rotational energies, Fig. 1b shows the iso-probability contour plot obtained from Eq. (11). The peak is seen to arise at a vibrational energy of $2347 \mathrm{~cm}^{-1}$, and a rotational energy of $2208 \mathrm{~cm}^{-1}$, which corresponds to the $\left(v^{\prime}=1, j^{\prime}=39\right)$ state of the oxygen molecule; see also panel (b) of Fig. 2.

Panel (a) of Fig. 2 compares the vibrational distributions obtained from the MGB scheme here proposed with those obtained from the traditional QCT method. As expected the results show only small differences, which may be explained from the large number of populated vibrational states. Significant differences arise though in the corresponding vibrationally-specific rotational distributions. This is illustrated in panel (b) of Fig. 2 which corresponds to the maximum populated vibrational state $\left(v^{\prime}=1\right)$. This is due to two major factors. First, the number of populated high-rotational states predicted from the traditional semi-classical quantization scheme is much larger than the true number of quantum mechanical rotational states that are obtained by solving the vibrational-rotational Schrödinger equation. By checking the maximum rotational state for each of the $v^{\prime}=0-46$ vibrational states calculated for $\mathrm{O}_{2}$, we have found that a total of 47 levels lie
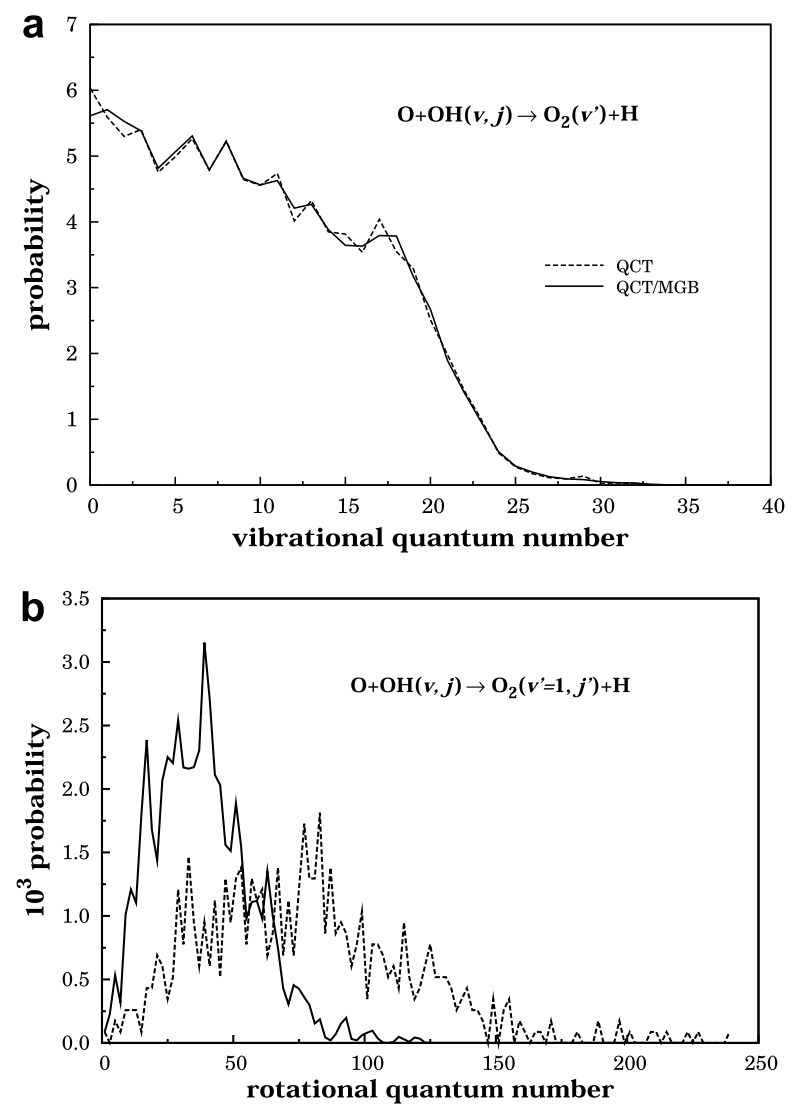

Fig. 2. Panel (a) shows the total vibrational distribution for product $\mathrm{O}_{2}$ in the $\mathrm{O}+\mathrm{OH}\left(v^{\prime}, j^{\prime}\right)$ reaction under LTD: dashed line, QCT; solid line, QCT/MGB. A similar comparison but for the rotational distribution of the most populated $\mathrm{O}_{2}$ vibrational state $\left(v^{\prime}=1\right)$ is in panel (b). higher than the highest QM rotational state obtained for their corresponding $v^{\prime}$ state. For example, one gets $j_{\max }^{\prime}=241$ for $v^{\prime}=0$ from the semi-classical analysis, thus exceeding the maximum rotational quantum number of $j_{\max }^{\prime}=235$ obtained quantum mechanically for the $\mathrm{O}_{2}$ potential curve of the $\mathrm{HO}_{2}$ DMBE IV PES. Similarly, $254 \mathrm{O}_{2}$ vibrational quantum numbers assigned in traditional QCT lie above the maximum quantum number of $v^{\prime}=46$. Note that many of the high-rotational states correspond to a physical states that violate the quantum mechanical ZPE, in a total of 356. It should be emphasized that the average uncertainty in the rotational energy for the above 47 missing high $-j^{\prime}$ states amounts to $28 \%$. Besides being due to the semi-classical nature of the rotational quantum number assignment, such a result reflects the difficulty in partitioning the internal energy into its components for high energy regimes. Unfortunately, to or knowledge, no accurate quantum mechanical rotational distribution is available in DMBE IV (or any other PES) for comparison. Finally, all the above remarks but one referring to violation of the ZPE criterion (and this to an extent of $\sim 0.4 \%$ ) should have no implication in the rate constants and major conclusions reported elsewhere [10].
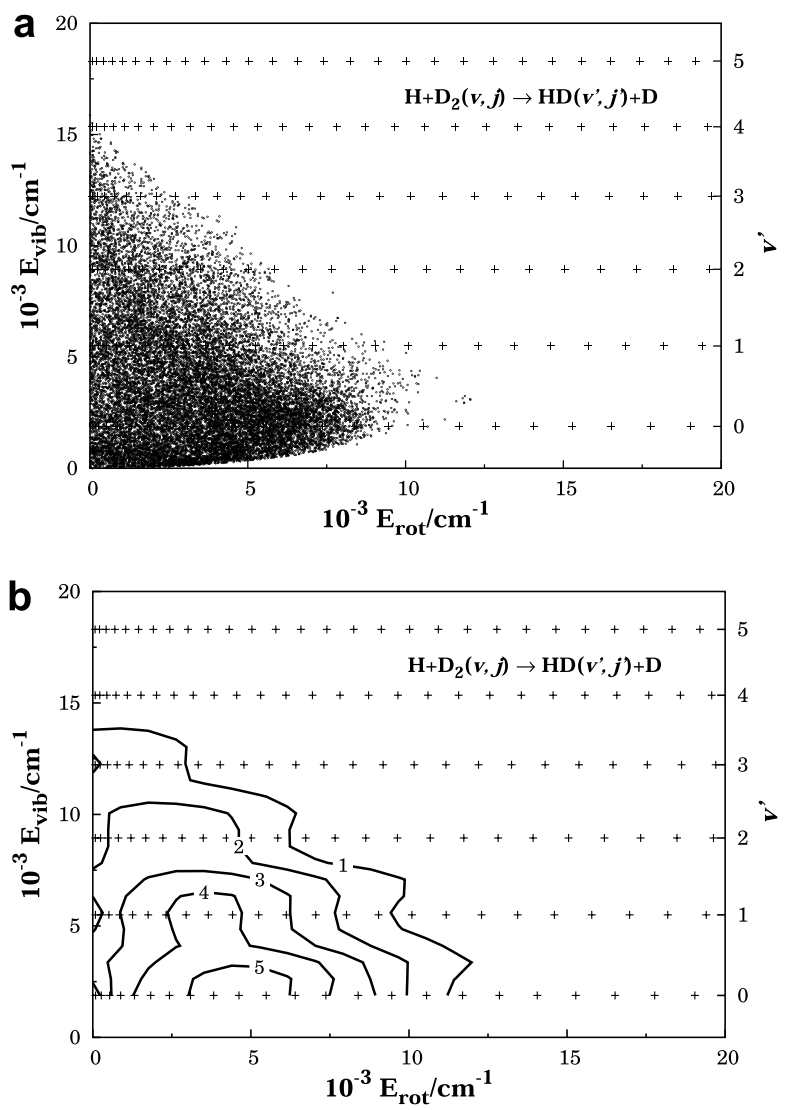

Fig. 3. As in Fig. 1 but for the product HD in the $\mathrm{H}+\mathrm{D}_{2}\left(v^{\prime}=0, j^{\prime}=0\right)$ reaction at a collisional energy of $1.85 \mathrm{eV}$. The 'pluses' indicate the 'exact' vibrational-rotational states of HD, while the traditional QCT results (all calculated values are shown) are indicated by the dots. Panel (b) shows isoprobability contours defined by $P\left(n_{\mathrm{c}}\right)=0.002 n_{\mathrm{c}}^{2}$, where $n_{\mathrm{c}}=1,2, \ldots$ (increasing inwards) indicates the contour level. 


\section{2. $\mathrm{H}+\mathrm{D}_{2}$ exchange reaction}

The title exchange reaction and other isotopic variants are popular prototypes of direct collision processes. They have been widely studied since the early days of reaction dynamics [17], with very good agreement between calculated and observed attributes, namely total reaction probabilities, state-to-state integral and differential cross sections, and thermal rate constant [18-29]. Such advances paralleled those in the construction of accurate PESs [30-33] for $\mathrm{H}_{3}$. However, most such studies concentrated at relatively low energies, and the theoretical ones restricted to the ground adiabatic PES. For high energies, Pomerantz et al. [34] reported a disagreement between theory and experiment in the product rotational distributions for the reaction $\mathrm{H}+\mathrm{D}_{2}(v=0, j=0) \rightarrow \mathrm{HD}\left(v^{\prime}=3, j^{\prime}\right)+\mathrm{D} . \quad$ Specifically, they have observed a warming at high $j^{\prime}$ states in the rotational distributions that could not be explained from accurate quantum calculations on the lowest sheet of the $\mathrm{H}_{3}$ PES (this shows a conical intersection between the ground and first-excited electronic states). Recently [35], we have carried out both single-surface (adiabatic) and two-surface diabatic quantum mechanical wave-packet calculations of this reaction. Despite being quite accurate, they too largely failed to support the Pomerantz et al. [34] results. Rather than focusing on this discrepancy, the calculations here reported are mainly intended to compare the product vibrational-rotational distributions obtained from the QCT/MGB method from the present work with the accurate quantum wavepacket ones [35]. Also shown for comparison are the results from the traditional QCT method and its QCT/VEQMT [14] (or simply VEQMT) variant, where in its simplest form all trajectories that form products with a déficit of ZPE content are discarded from the statistical analysis. Thus, only the lowest sheet of the popular DMBE PES [31] has been employed. Of the energies considered in Ref. [35], we have chosen here the largest, $E_{\mathrm{tr}}=1.85 \mathrm{eV}$.

Shown in panel (a) of Fig. 3 are the results of the 19947 reactive QCT trajectories superimposed on the calculated

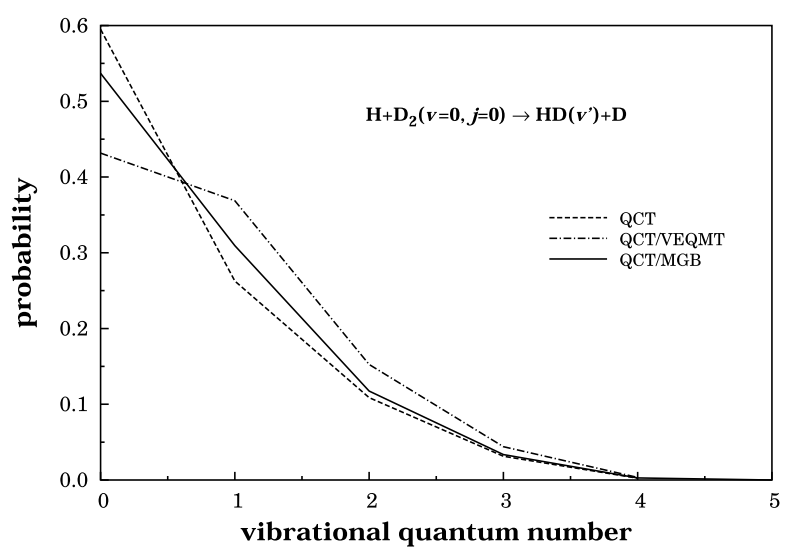

Fig. 4. Total product vibrational distribution for the reaction $\mathrm{H}+\mathrm{D}_{2}\left(v^{\prime}=0, j^{\prime}=0\right) \rightarrow \mathrm{HD}\left(v^{\prime \prime}, j^{\prime \prime}\right)+\mathrm{D}$ at a collisional energy of $1.85 \mathrm{eV}$ : dashed line, QCT; dash-dot line, QCT/VEQMT; solid QCT/ MGB.
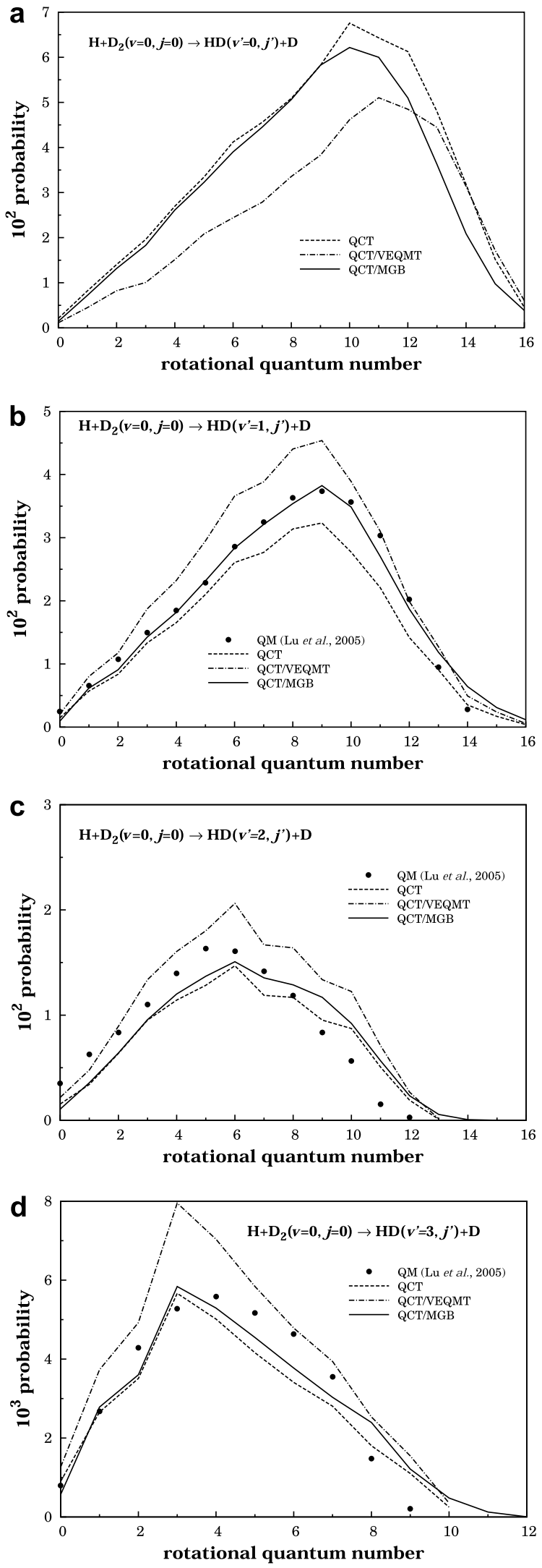

Fig. 5. Product rotational distributions in the reaction $\mathrm{H}+\mathrm{D}_{2}(v=0$, $j=0) \rightarrow \mathrm{HD}\left(v^{\prime}, j^{\prime}\right)+\mathrm{D}$, with panels (a) to (d) for $v^{\prime}=0-3$, respectively. Key: dashed line, QCT; dash-dot line, QCT/VEQMT; solid line, QCT/ MGB. Also shown by the solid dots are the accurate quantum wavepacket results [35] for $v^{\prime \prime}=1-3$. 
quantum mechanical grid of vibrational-rotational eigenvalues as obtained for the $\mathrm{H}_{2}$ curve in the $\mathrm{H}_{3}$ DMBE PES. In turn, Fig. 3b shows the iso-probability contour plot obtained from the new MGB scheme of the present work. The notable feature is perhaps the large number of HD molecules (5737) that end up with a vibrational content below ZPE. Such trajectories are in the VEQMT [14] method considered a physical, and discarded from the statistical analysis. Being a large fraction $(29 \%)$ of total number of trajectories run, it is not surprising that VEQMT leads to significant differences in the vibrational and rotational distributions shown in Figs. 4 and 5. Also notable is the good agreement between the MGB results and the traditional QCT ones. As might be anticipated, the decrease in total reactive probability for $v^{\prime}=0$ due to ZPE leakage is now significantly smaller than in VEQMT, with the current MGB results lying somewhere between the QCT and VEQMT ones. In fact, the number of trajectories with $j^{\prime} \geqslant 9$ that lack ZPE content amounts to 2362 while the error in the rotational energy due to the vibrationalrotational partition scheme is $12.3 \%$. Such a high percent of high $j^{\prime}$ trajectories that lack ZPE content is largely due to a flow of vibrational into rotational energy, and may largely explain the differences observed between the QCT and MGB rotational distributions for specific vibrational states. A final remark to note the good agreement between the rotational distributions from the present work and the time-dependent quantum mechanical ones [35] for $v^{\prime}=1$, and to a smaller extent for other $v^{\prime}$ states (particularly $v^{\prime}=2$, where a warming of higher rotational states is observed in QCT, MGB, and VEQMT, by increasing order). Except for $v^{\prime}=0$ where a significant reduction is observed, the VEQMT method tends to overshoot the accurate quantum distributions due to discarding a significant fraction of trajectories from the statistical analysis. Finally, although not shown for brevity, all methods predict the $v^{\prime}=4$ state to be open but with a relative probability about a factor of eight smaller than for $v^{\prime}=3$.

\section{Concluding remarks}

We have suggested a simple scheme to 'quantize' the continuous vibrational-rotational energy distributions obtained from QCT calculations. The new MGB approach has been applied to the much studied $\mathrm{O}+\mathrm{OH}$ and $\mathrm{H}+\mathrm{D}_{2}$ reactions with promising results. As major features, we emphasize the following: (a) the $\rho_{\alpha}$ and $\rho_{\beta}$ parameters in it are fixed a priori without resorting to any fit of dynamics data; (b) the method accounts approximately for ZPE leakage without discarding any of the calculated trajectories (thus, without disturbing [6] the QCT statistics); (c) by introducing two cardinal numbers that identify the sets of vibrational and rotational quantum numbers, one may conjecture that it is generalizable to reactions with larger product molecules where the assignment of quantum numbers from classical techniques has not been possible thus far. Clearly, further developments and applications are required to assess its merit.

\section{Acknowledgement}

This work has been supported by Fundação para a Ciência e Tecnologia, Portugal under contracts POCI/ QUI/60501/2004, POCI/AMB/60261/2004, and REEQ/ 128/QUI/2005.

\section{References}

[1] L. Bonnet, J.C. Rayez, Chem. Phys. Lett. 277 (1997) 183.

[2] L. Bonnet, J.C. Rayez, Chem. Phys. Lett. 397 (2004) 106.

[3] L. Bañares, F. Aoiz, P. Honvault, B. Bussery-Honvault, J.M. Launay, J. Chem. Phys. 118 (2003) 565.

[4] A.J.C. Varandas, Int. Rev. Phys. Chem. 19 (2000) 199.

[5] D. Rapp, Quantum Mechanics, Holt, Rinehart and Winston Inc., New York, 1971.

[6] A.J.C. Varandas, Chem. Phys. Lett. 225 (1994) 18.

[7] A.J.C. Varandas, L. Zhang, Chem. Phys. Lett. 340 (2001) 62

[8] W.L. Hase et al., QCPE Bull. 16 (1996) 43.

[9] A.J.C. Varandas, in: D.R. Yarkony, H. Köppel, W. Domcke (Eds.), Electronic Structure, Spectroscopy and Dynamics, Advanced Series in Physical Chemistry, World Scientific Publishing, 2004, p. 91 (Chapter 5).

[10] A.J.C. Varandas, Chem. Phys. Lett. 396 (2004) 182.

[11] S. Adler-Golden, J. Geophys. Res. 102 (1997) 19969.

[12] A.J.C. Varandas, ChemPhysChem 6 (2005) 453.

[13] M.R. Pastrana, L.A.M. Quintales, J. Brandão, A.J.C. Varandas, J. Phys. Chem. 94 (1990) 8073.

[14] A.J.C. Varandas, J. Chem. Phys. 99 (1993) 1076.

[15] A.J.C. Varandas, J. Phys. Chem. A 107 (2003) 3769.

[16] J.R. Le Roy, Level 7.5: A Computer Program for Solving the Radial Schrödinger Equation for Bound and Quasibound Levels, Tech. Rep. CP-655, University of Waterloo, Chemical Physics Research Report, 2002.

[17] F. Fernández-Alonso, R.N. Zare, Annu. Rev. Phys. Chem. 53 (2002) 67.

[18] S. Sukiasyan, H.D. Meyer, J. Chem. Phys. 116 (2002) 10641.

[19] D.E. Manolopoulos, R.E. Wyatt, J. Chem. Phys. 92 (1990) 810.

[20] J. Chang, N.J. Brown, J. Chem. Phys. 103 (1995) 4097.

[21] I.L.D.M. Charutz, M. Baer, J. Chem. Phys. 106 (1997) 6160.

[22] L. Bañares et al., J. Chem. Phys. 108 (1998) 6160

[23] B.K. Kendrick, J. Chem. Phys. 114 (2001) 8796.

[24] B.D. Bean, J.D. Ayers, F. Fernández-Alonso, R.N. Zare, J. Chem. Phys. 116 (2002) 6634.

[25] S.C. Althorpe, J. Chem. Phys. 117 (2002) 4623.

[26] S.A. Harich, D. Dai, C.C. Wang, X. Yang, S.D. Chao, R.T. Skodje, Nature 419 (2002) 281.

[27] S.L. Mielke et al., Phys. Rev. Lett. 91 (2003) 063201.

[28] D.X. Dai, C.C. Wang, S.A. Harich, X.Y. Wang, X.M. Yang, S.D. Chao, R.T. Skodje, Science 300 (2003) 1730.

[29] F. Ausfelder, A.E. Pomerantz, R.N. Zare, S.C. Althorpe, F.J. Aoiz, L. Bañares, J.F. Castillo, Science 120 (2004) 3255.

[30] D.G. Truhlar, C.J. Horowitz, J. Chem. Phys. 68 (1978) 2466

[31] A.J.C. Varandas, F.B. Brown, C.A. Mead, D.G. Truhlar, N.C. Blais, J. Chem. Phys. 86 (1987) 6258.

[32] A.I. Boothroyd, W.J. Keogh, P.G. Martin, M.R. Peterson, J. Chem. Phys. 95 (1991) 4343.

[33] R. Abrol, A. Shaw, A. Kuppermann, D.R. Yarkony, J. Chem. Phys. 115 (2001) 4640 .

[34] A.E. Pomerantz, F. Ausfelder, R.N. Zare, S.C. Althorpe, F.J. Aoiz, L. Bañares, J.F. Castillo, J. Chem. Phys. 120 (2004) 3244.

[35] R.F. Lu, T.S. Chu, Y. Zhang, K. Han, A.J.C. Varandas, J.Z.H Zhang, J. Chem. Phys. 125 (2006) 133108. 